Research Article

\title{
Quality Analysis of Long dan Xie gan Pill by a Combination of Fingerprint and Multicomponent Quantification with Chemometrics Analysis
}

\author{
Jing Liu $\mathbb{D}^{1},{ }^{1}$ Hui Liu, ${ }^{1,2}$ Zhong Dai $\mathbb{D}^{1},{ }^{1}$ and Shuangcheng Ma ${ }^{1}{ }^{1}$ \\ ${ }^{1}$ National Institutes for Food and Drug Control, Beijing 100050, China \\ ${ }^{2}$ Institute of Food and Drug, Yanbian Korean Autonomous Prefecture, Jilin Province 133002, China \\ Correspondence should be addressed to Zhong Dai; daizhong@nifdc.org.cn and Shuangcheng Ma; masc@nifdc.org.cn
}

Jing Liu and Hui Liu contributed equally to this work.

Received 17 July 2018; Revised 17 October 2018; Accepted 21 November 2018; Published 11 December 2018

Academic Editor: Ricardo Jorgensen Cassella Copyright $\odot 2018$ Jing Liu et al. This is an open access article distributed under the Creative Commons Attribution License, which
permits unrestricted use, distribution, and reproduction in any medium, provided the original work is properly cited.

\begin{abstract}
Long dan Xie gan pill is a traditional complex compound preparation with a long history for treatment of diseases, including hepatocolic hygropyrexia, dizziness, tinnitus, and deafness. Quality of products from different manufacturers may be varied. Since the current standard could not control the quality of products in a comprehensive and effective way, this study aimed at establishing a practical and convenient approach for holistic quality control of the preparation. This study included both qualitative and quantitative works to get information on the overall composition and main components, respectively. As a result, HPLC fingerprint (UV $240 \mathrm{~nm}$ ) similarities of all fifty samples were in the range of $0.65 \sim 0.99$. Results indicated that there was a difference among products from different manufacturers. Additionally, ten characteristic peaks of the fingerprint were tentatively identified by LC-MS. Further chemometrics analysis was utilized to evaluate the products from different manufacturers. At the same time, the HPLC (UV 285 nm) multicomponent quantification result showed that contents of gentiopicrin, baicalin, baicalein, and wogonin were in the range of $0.61-5.40,1.96-5.33,0.10-3.40$, and $0.046-1.16 \mathrm{mg} \cdot \mathrm{g}^{-1}$, respectively. Data analysis verified the main different component of baicalein from the fingerprint statistical analysis. It is worth mentioning that the qualitative fingerprint and quantitative multicomponent determination were simultaneously accomplished by HPLC-DAD with dual channels. The study provided sound basis for improving quality control standards. This study also provided practical strategy for overall quality control of traditional Chinese medicines.
\end{abstract}

\section{Introduction}

Long dan Xie gan pill is prepared from ten species of crude drugs including Gentianae radix et rhizoma, Scutellariae radix, and Akebiae caulis (Mutong) in Chinese Pharmacopoeia (2015 Edition, Volume I) [1]. It is widely used for the treatment of diseases, including hepatocolic hygropyrexia, dizziness, tinnitus and deafness, hypochondriac pain [1]. The preparation has attracted widespread attention, since it caused aristolochic acid nephropathy (AAN) $[2,3]$. At that time, the prescription collected the crude drug of Caulis aristolochiae manshuriensis (Guanmutong) instead of Akebiae caulis [4-6]. Because of the serious adverse effect of aristolochic acids, the medicinal standard of Caulis aristolochiae manshuriensis was abolished and replaced by Akebiae caulis without containing such toxic constituents since 2003.

As one commonly used Chinese patent medicine (CPM) with a long history, Long dan Xie gan pill has about 200 manufacturers. Therefore, the quality of products from different manufacturers may be varied. Since quality is directly related to drug safety and efficacy, it is very important to evaluate the holistic quality of the products. Researchers have been working on the essential quality control and evaluation methods for years [2]. For most CPMs, the effective components are not clear and the consistency of product quality is a key indicator of quality product evaluation. It is worth mentioning that fingerprint is an internationally recognized effective method because it could reflect the overall quality information [2-10]. However, 
fingerprint is usually used for qualitative consistency evaluation. And the quantification could be achieved by applying multicomponent determination [10-13]. In recent years, more and more chromatographic and spectroscopic methods including LC, LC-MS ${ }^{\mathrm{n}}$, and quantitative nuclear magnetic resonance (QNMR) are applied for the aforementioned qualitative and quantitative work [14-21]. Among these methods, high-performance liquid chromatography (HPLC) is still the main method deployed in quality control of traditional Chinese medicines (TCMs) because of its advantages, including good repeatability, wide application, and high efficiency.

In this study, the qualitative and quantitative consistency information of Long dan Xie gan pill samples was achieved at the same time by a combination of fingerprint with multicomponent quantification by HPLC-diode array detector (DAD) with dual channels (UV $240 \mathrm{~nm}$ and $285 \mathrm{~nm}$ ). Additionally, further deep mining of the data by chemometrics analysis helped to evaluate the differences of products in a more comprehensive and effective way. The results indicated that the established method could comprehensively analyze the product quality. This strategy could provide a practical approach for the holistic quality control of TCM.

\section{Materials and Methods}

2.1. Chemicals and Reagents. Gentiopicrin (97.6\%, batch no. 110770-201716), baicalin (93.5\%, batch no. 110715-201720), baicalein (98.5\%, batch no. 111595-201607), and wogonin (100\%, batch no. 111514-201605) were from the National Institutes for Food and Drug Control, Beijing, China. Methanol (analytical reagent) was from National Drug Chemical Reagents Co. Ltd. Acetonitrile (chromatographic pure) and formic acid (mass spectrometry reagent) were from Thermo Fischer Scientific. The water was of ultrahigh purity.

2.2. Materials. Fifty batches of Long dan Xie gan pill samples were from 8 manufacturers $(\mathrm{A} \sim \mathrm{H})$. All the samples involve two dosage forms including water-bindered pills (WBP) and big candied pills (BCP). The detailed information is listed as follows (Table 1).

2.3. Instrumentations. HPLC analysis was performed on a Waters 2690 HPLC instrument (Waters, Milford, USA), equipped with a DAD, an autosampler and a column heater. METTLER XS105 electronic analytical balance (Mettler-toledo, Zurich, Switzerland), Milli-Q water purification system (Milli-pore, Burlington, USA), and KQ300DA numerical control ultrasound cleaning instrument (Kunshan Ultrasonic Instruments Co. Ltd., Kunshan, China) were used. Chemometrics analysis was achieved by ChemPattern software (Chenmind Technologies Co., Ltd., Beijing, China).
TABLE 1: Sample information.

\begin{tabular}{|c|c|c|c|}
\hline No. & Manufacturers & Batch number & Dosage \\
\hline 1 & \multirow{10}{*}{ A } & B16099 & \multirow{5}{*}{ WBP } \\
\hline 2 & & B17001 & \\
\hline 3 & & B17045 & \\
\hline 4 & & B16033 & \\
\hline 5 & & B16040 & \\
\hline 6 & & A17057 & \multirow{5}{*}{$\mathrm{BCP}$} \\
\hline 7 & & A16065 & \\
\hline 8 & & A16119 & \\
\hline 9 & & A17004 & \\
\hline 10 & & A17104 & \\
\hline 11 & \multirow{5}{*}{ B } & 20170502 & \multirow{5}{*}{ WBP } \\
\hline 12 & & 20170504 & \\
\hline 13 & & 20170503 & \\
\hline 14 & & 171002 & \\
\hline 15 & & 171003 & \\
\hline 16 & \multirow{5}{*}{$\mathrm{C}$} & 1704058 & \multirow{5}{*}{ WBP } \\
\hline 17 & & 1801024 & \\
\hline 18 & & 1801027 & \\
\hline 19 & & 1801026 & \\
\hline 20 & & 1711016 & \\
\hline 21 & \multirow{10}{*}{$\mathrm{D}$} & 20180336 & \multirow{5}{*}{ WBP } \\
\hline 22 & & 20180335 & \\
\hline 23 & & 20180334 & \\
\hline 24 & & 20180452 & \\
\hline 25 & & 20180451 & \\
\hline 26 & & 20170621 & \multirow{5}{*}{$\mathrm{BCP}$} \\
\hline 27 & & 20180419 & \\
\hline 28 & & 20170620 & \\
\hline 29 & & 20170504 & \\
\hline 30 & & 20170308 & \\
\hline 31 & \multirow{5}{*}{$\mathrm{E}$} & 180203 & \multirow{5}{*}{ WBP } \\
\hline 32 & & 180201 & \\
\hline 33 & & 180204 & \\
\hline 34 & & 180205 & \\
\hline 35 & & 180202 & \\
\hline 36 & \multirow{5}{*}{$\mathrm{F}$} & 171102 & \multirow{5}{*}{ WBP } \\
\hline 37 & & 171002 & \\
\hline 38 & & 170902 & \\
\hline 39 & & 171101 & \\
\hline 40 & & 171001 & \\
\hline 41 & \multirow{5}{*}{ G } & 1801001 & \multirow{5}{*}{ WBP } \\
\hline 42 & & 1802005 & \\
\hline 43 & & 1801003 & \\
\hline 44 & & 1801004 & \\
\hline 45 & & 1802006 & \\
\hline 46 & \multirow{5}{*}{$\mathrm{H}$} & 180102 & \multirow{5}{*}{$\mathrm{BCP}$} \\
\hline 47 & & 180101 & \\
\hline 48 & & 171001 & \\
\hline 49 & & 171002 & \\
\hline 50 & & 160501 & \\
\hline
\end{tabular}

2.4. Preparation of Standard Solutions. Standard stock solutions of baicalin $\left(0.1 \mathrm{mg} \cdot \mathrm{mL}^{-1}\right)$ were prepared by dissolving suitable amounts of reference substance in methanol for fingerprint establishment.

Standard stock mixed solution of gentiopicrin $\left(0.4972 \mathrm{mg} \cdot \mathrm{mL}^{-1}\right)$, baicalin $\left(0.5268 \mathrm{mg} \cdot \mathrm{mL}^{-1}\right)$, baicalein $\left(0.4688 \mathrm{mg} \cdot \mathrm{mL}^{-1}\right)$, and wogonin $\left(0.5080 \mathrm{mg} \cdot \mathrm{mL}^{-1}\right)$ was 
prepared by dissolving suitable amounts of each reference substance in methanol for multicomponents assay.

2.5. Preparation of Sample Solutions. For Long dan Xie gan pill (WBP) (6g per small bag), 5 bags were mixed and pulverized to powder. For Long dan Xie gan pill (BCP) $(6 \mathrm{~g}$ per pill), 5 pills were cut into small pieces. Then, $2 \mathrm{~g}$ were weighed accurately and put into a $50 \mathrm{~mL}$ plug conical bottle. Twenty-five $\mathrm{mL}$ methanol (for WBP samples) and $25 \mathrm{~mL}$ $80 \%$ methanol-water solution (for BCP samples) were added precisely and weighed, respectively. After extracting by ultrasonication (power: $300 \mathrm{~W}$; frequency: $40 \mathrm{kHz}$ ) for $30 \mathrm{~min}$, the extract was cooled down and then made up for lost weight by adding methanol (for WBP samples) or $80 \%$ methanol (for BCP samples). The continuous filtrate was taken and then filtered by $0.22 \mu \mathrm{m}$ microporous filter membrane.

2.6. HPLC-DAD Chromatographic Condition. Column: Phenomenex Gemini C18 $(4.60 \times 250 \mathrm{~mm}, 5 \mu \mathrm{m})$; mobile phase: gradient elution with acetonitrile- (A-) $0.1 \%$ formic acid-water solution (B) (0-5 min, 7\% A-10\%A; 5-11 min, $10 \% \mathrm{~A}-15 \% \mathrm{~A} ; 11-15 \mathrm{~min}, 15 \% \mathrm{~A}-20 \% \mathrm{~A} ; 15-32 \mathrm{~min}, 20 \%$ A-30\%A; 32-54 min, 30\%A-55\%A; 54-60 min, 55\%A-80\% $\mathrm{A}$; and $60-68 \mathrm{~min}, 7 \% \mathrm{~A}$ ); flow rate: $1.0 \mathrm{~mL} \cdot \mathrm{min}^{-1}$; column temperature: $30^{\circ} \mathrm{C}$; injection volume: $10 \mu \mathrm{L}$; detection wavelength: UV $240 \mathrm{~nm}$ for fingerprint and UV $285 \mathrm{~nm}$ for multicomponent determination. The typical chromatograms were shown as Figures 1 and 2, respectively.

2.7. Mass Spectrometry Condition. MS analysis was performed on an Agilent1260-6410B LC-MS couplet system equipped with Agilent Mass Hunter ChemStation (Agilent, Santa Clara, USA). The mass spectrometry settings were as follows: split ratio $=1: 9$; desolvation temperature: $350^{\circ} \mathrm{C}$; desolvation air flow $\mathrm{N}_{2}: 540 \mathrm{~L} \cdot \mathrm{h}^{-1}$; nebulizer pressure: $30 \mathrm{psi}$; and capillary: $4000 \mathrm{~V}$. Both positive and negative modes were performed with a scan range of $\mathrm{m} / z$ 50-1200.

\section{Results and Discussion}

3.1. Results of Fingerprint. For fingerprint study, all the samples were prepared and analyzed according to conditions under 2.5 and 2.6. Baicalin $\left(t_{\mathrm{R}}=34.02 \mathrm{~min}\right)$ was taken as the reference peak. Relative retention times (RRTs) and relative peak areas (RPAs) of the characteristic peaks were calculated for method validation.

3.1.1. Instrument Precision. The same sample solution (no. 21) was injected for six consecutive times. The result showed that the RSDs of RRTs and RPAs were in the range of $0.073 \%-2.0 \%$ and $0.26-1.74 \%$, respectively. It showed that the precision of the instrument was good.

3.1.2. Repeatability. The same batch sample (no. 21) was taken and prepared for six independent sample solutions for analysis. The result showed that the RSDs of RRT and
RPA were in the range of $0.010 \%-0.44 \%$ and $0.51-2.24 \%$, respectively. It indicated that method repeatability was good.

3.1.3. Stability. The same sample solution (no. 21) was injected at $0,4,8,12,16,20$, and $24 \mathrm{~h}$ at room temperature. The result showed that the RSDs of RRT and RPA were in the range of $0.039 \%-0.81 \%$ and $0.71-4.58 \%$, respectively. It demonstrated the sample solution was stable within $24 \mathrm{~h}$.

3.1.4. Establishment of Fingerprint. After fifty batches of the sample solutions were analyzed, their chromatograms (UV $240 \mathrm{~nm}$ ) were recorded (Figure 3) and imported to ChemPattern software. All variables were used as the common peak screening condition. And Gauss curve simulation method was applied to generate the common mode with 16 characteristic peaks (Figure 4). All sample chromatograms were analyzed by comparison with the common mode.

3.1.5. Identification and Attribution of Characteristic Peaks. The sample solution was analyzed according to the conditions under Sections 2.6 and 2.7. By combination with the chromatographic behavior of the components, ten characteristic peaks were identified by comparing with reference standards. Also the main origins of the peaks were attributed (Table 2), and they were mainly from five species of crude drugs in the prescription.

\subsubsection{Statistical Analysis}

(1) Similarity Analysis. The above HPLC (UV $240 \mathrm{~nm}$ ) fingerprint common mode was taken as a reference. During the analysis, the included angle cosine method was used to calculate the similarity of each sample (Figure 5). Finally, the similarities of all samples were in the range of $0.65 \sim 0.99$. Among them, similarities of seven batches of samples were lower than 0.8 , including all samples from enterprise B. The result indicated that there was a certain difference among the overall product quality of these samples from others. Also it was clear that the uniformity of most BCP samples (no. 6 10 and 26 30) was not good as the WBP ones.

(2) Principle Component Analysis. Principle component analysis (PCA) was carried out after standardization of all sample data (Figures 6 and 7). The contribution rates of the first and second principle component (PC1) were $45.92 \%$ and $39.22 \%$, respectively. And the total contribution rate of $85.14 \%$ showed that it could reflect the differences between samples in a more comprehensive way. PCA scatter plot (Figure 6) displayed that samples from each enterprise basically could be grouped into a class. It showed that samples from enterprise B deviated far away from others. The principle component load diagram (Figure 7) gave the proportion of each chromatographic peak in the principal component. And the greater the distance from $X=0$ 


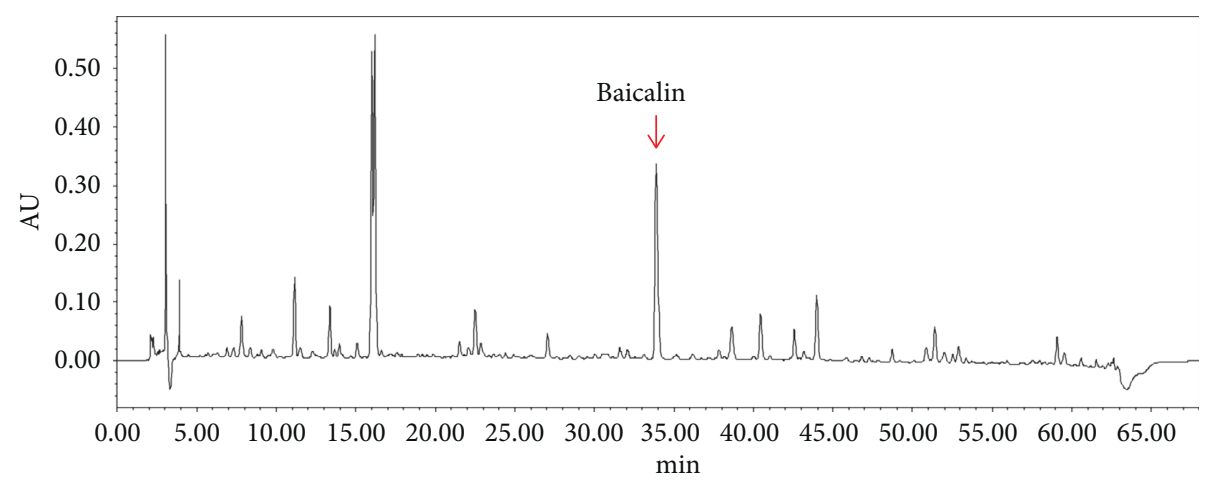

FIgURE 1: HPLC (UV $240 \mathrm{~nm}$ ) chromatogram of the typical sample.

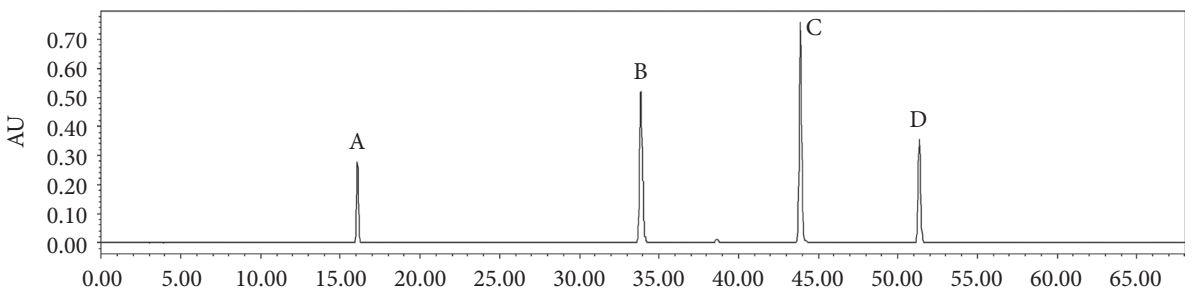

(a)

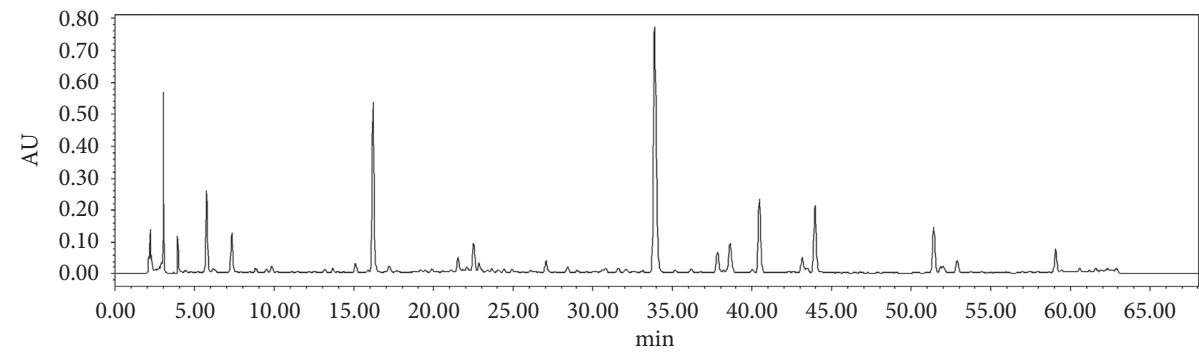

(b)

FIGURE 2: HPLC (UV $285 \mathrm{~nm}$ ) chromatograms of mixed standard solution (a) (A, Gentiopicrin; B, Baicalin; C, Baicalein; and D, Wogonin) and typical sample (b).

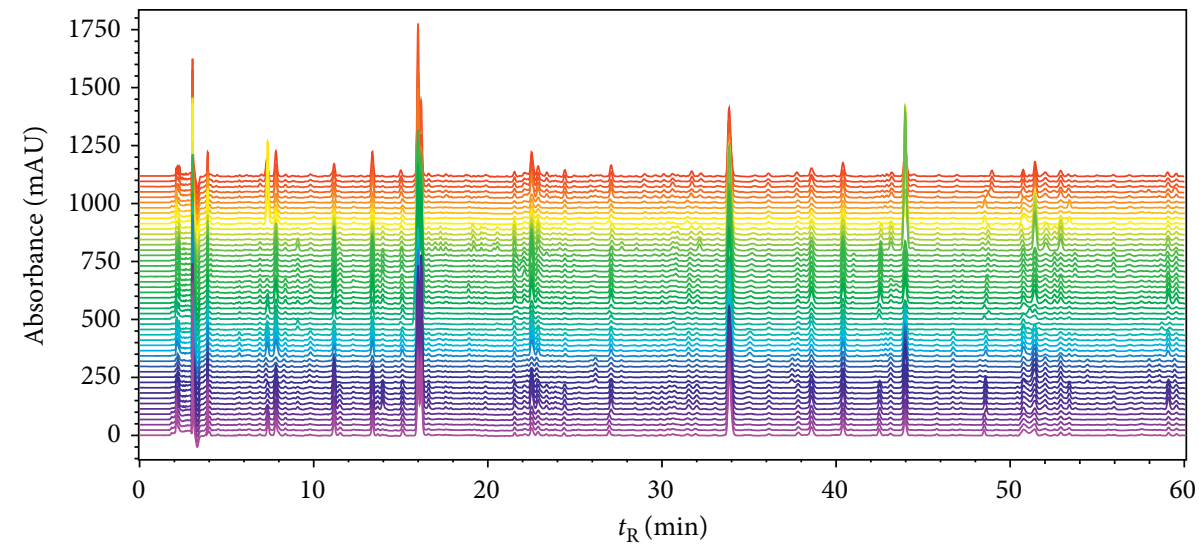

Figure 3: HPLC chromatograms (UV240 nm) of Long dan Xie gan pill samples.

longitudinal axis, the greater the contribution to PC1, such as gentiopicrin and baicalein. Likewise, the greater the distance from $Y=0$ transverse axis, the greater the contribution to PC2, such as baicalein and geniposide. The result displayed that samples from enterprise $\mathrm{B}$ separated with others along both PC1 and PC2. Therefore, the major 


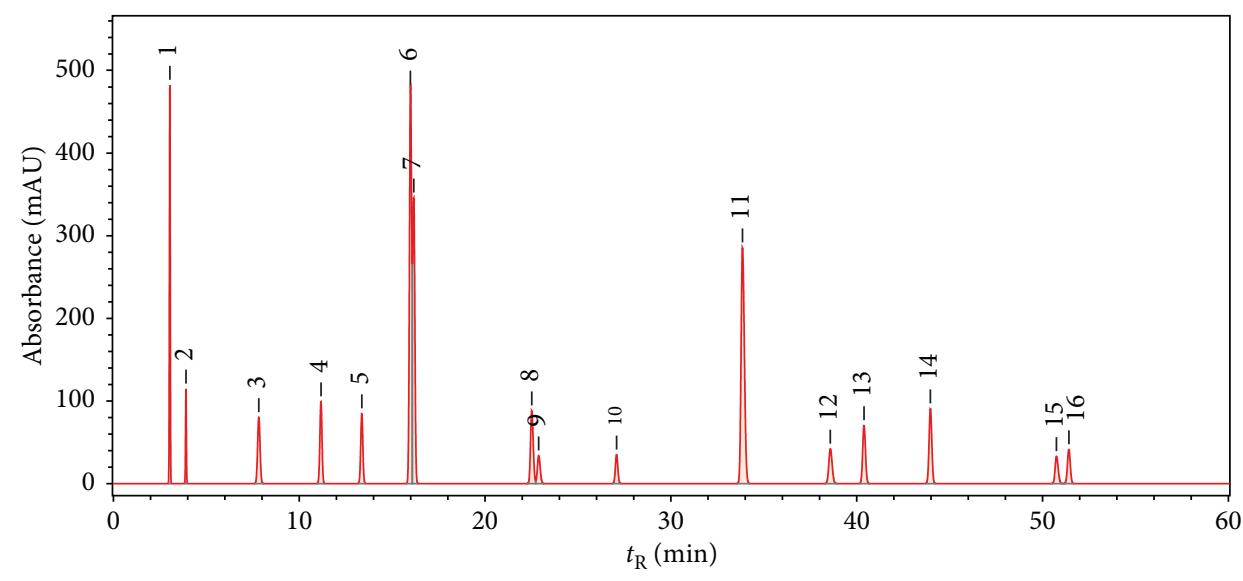

Figure 4: HPLC (UV240 nm) common mode of Long dan Xie gan pill samples.

TABLE 2: Identification of characteristic peaks of Long dan Xie gan pill.

\begin{tabular}{|c|c|c|c|c|c|c|}
\hline Peak no. & Compounds & $t_{\mathrm{R}}(\min )$ & Molecular formula & $\begin{array}{c}\text { Molecular } \\
\text { weight }\end{array}$ & $\begin{array}{c}\text { Quasimolecular } \\
\text { ions }\end{array}$ & Origin \\
\hline 3 & Geniposidic acid & 7.83 & $\mathrm{C}_{16} \mathrm{H}_{22} \mathrm{O}_{10}$ & 374.34 & $372.9[\mathrm{M}-\mathrm{H}]^{-}$ & Gardeniae fructus \\
\hline 6 & Geniposide & 15.07 & $\mathrm{C}_{17} \mathrm{H}_{24} \mathrm{O}_{10}$ & 388.37 & $433.0[\mathrm{M}+\mathrm{HCOO}]^{-}$ & Gardeniae fructus \\
\hline 7 & Gentiopicrin & 16.18 & $\mathrm{C}_{16} \mathrm{H}_{20} \mathrm{O}_{9}$ & 356.32 & $400.9[\mathrm{M}+\mathrm{HCOO}]^{-}$ & Gentianae radix et rhizoma \\
\hline 8 & Verbascoside & 22.86 & $\mathrm{C}_{29} \mathrm{H}_{36} \mathrm{O}_{15}$ & 624.59 & $623.0[\mathrm{M}-\mathrm{H}]^{-}$ & Plantaginis semen/rehmanniae radix \\
\hline 10 & Baicalin & 33.88 & $\mathrm{C}_{21} \mathrm{H}_{18} \mathrm{O}_{11}$ & 446.36 & $445.0[\mathrm{M}-\mathrm{H}]^{-}$ & Scutellariae radix \\
\hline 11 & Isomer of wogonoside & 38.62 & $\mathrm{C}_{22} \mathrm{H}_{20} \mathrm{O}_{11}$ & 460.39 & $459.0[\mathrm{M}-\mathrm{H}]^{-}$ & Scutellariae radix \\
\hline 12 & Wogonoside & 40.44 & $\mathrm{C}_{22} \mathrm{H}_{20} \mathrm{O}_{11}$ & 460.39 & $459.0[\mathrm{M}-\mathrm{H}]^{-}$ & Scutellariae radix \\
\hline 14 & Baicalein & 43.82 & $\mathrm{C}_{15} \mathrm{H}_{10} \mathrm{O}_{5}$ & 270.24 & $268.9[\mathrm{M}-\mathrm{H}]^{-}$ & Scutellariae radix \\
\hline 15 & Isomer of wogonin & 51.40 & $\mathrm{C}_{16} \mathrm{H}_{12} \mathrm{O}_{5}$ & 284.26 & $282.9[\mathrm{M}-\mathrm{H}]^{-}$ & Scutellariae radix \\
\hline 16 & Wogonin & 51.99 & $\mathrm{C}_{16} \mathrm{H}_{12} \mathrm{O}_{5}$ & 284.26 & $282.9[\mathrm{M}-\mathrm{H}]^{-}$ & Scutellariae radix \\
\hline
\end{tabular}

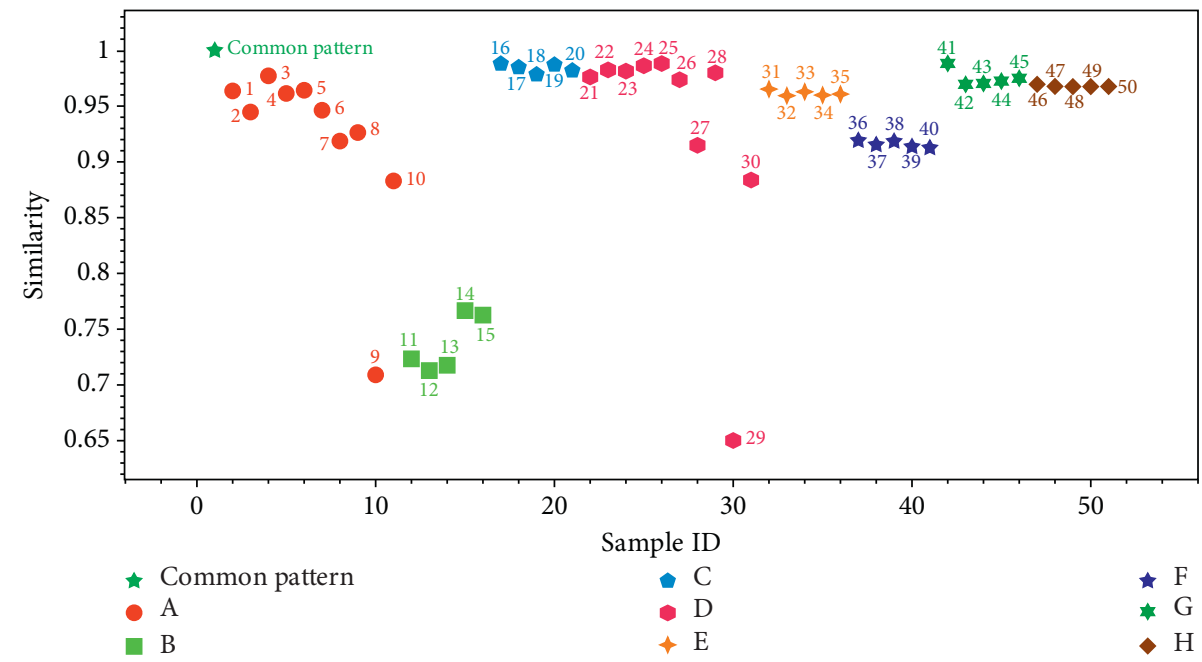

Figure 5: Similarity analysis results of Long dan Xie gan pill samples.

contributions to PC1 and PC2 were their main differential components from others. Because of the location at the position of PC1 $>0$ and PC2 $<0$, contents of such compounds were in positive correlation with $\mathrm{PC} 1$ and in negative correlation with PC2. Therefore, the contents of baicalein and wogonin were higher in these samples. Along the PC1, the concentration of baicalein distinguished the samples of enterprise $B$, whose values were higher than those presented for the others (Figure 6). On the contrary, along with the PC2, the levels of geniposide and gentiopicrin showed that there was a tendency for the separation of the products of enterprises D and $G$ from others. One sample of enterprise A was grouped with samples of enterprises D and G, which presented higher levels for these compounds. 


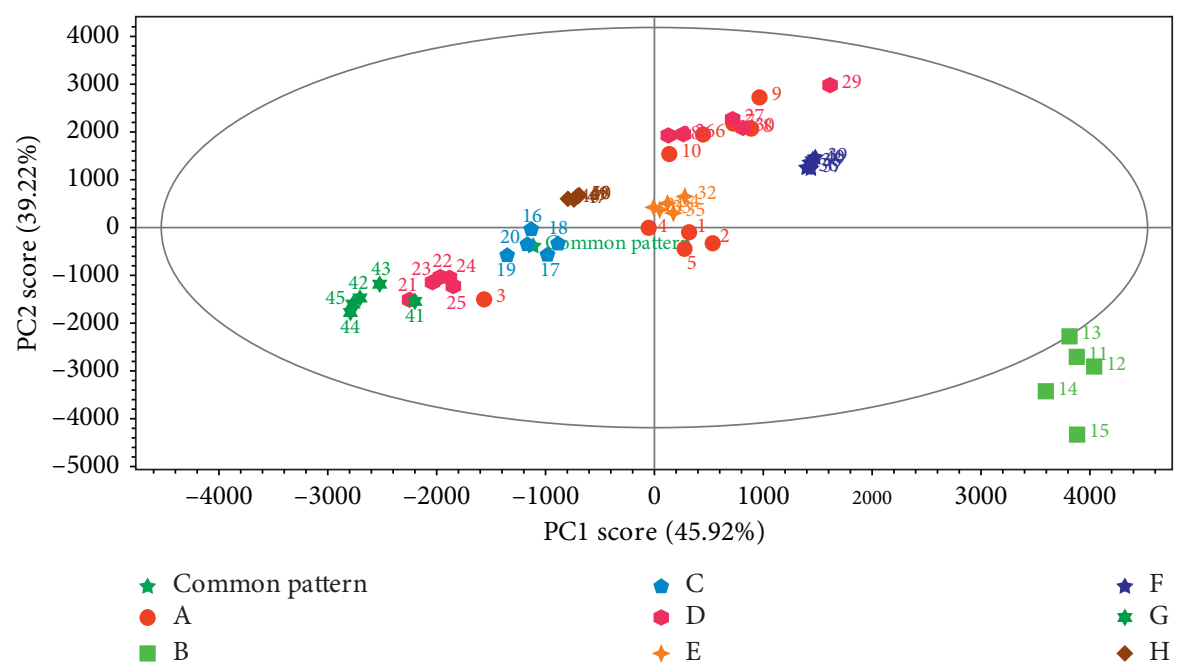

FIgURe 6: PCA score scatter plot of Long dan Xie gan pill samples.

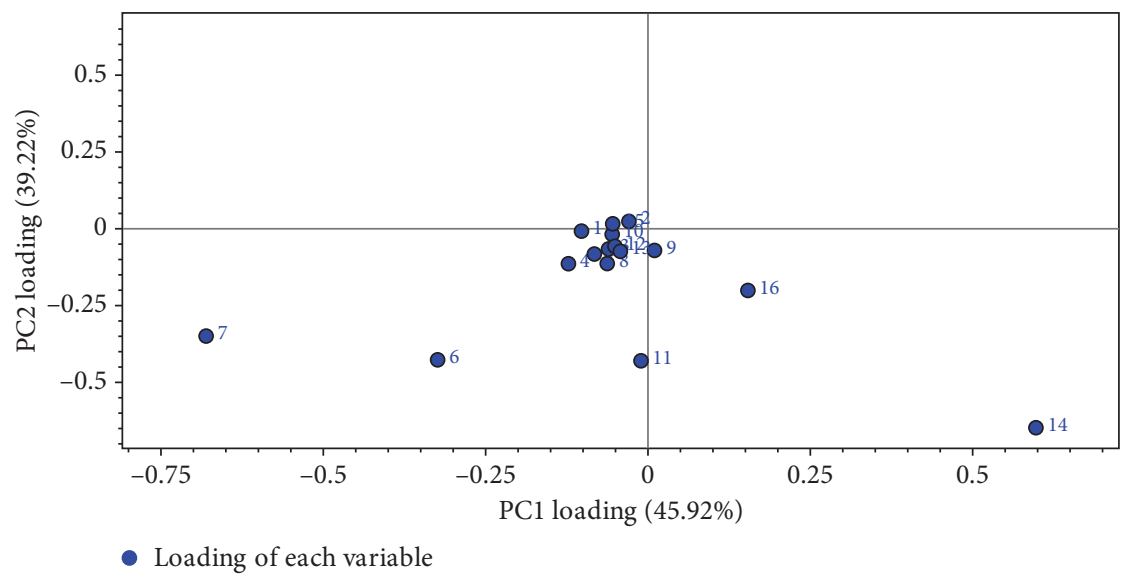

Figure 7: PCA loading scatter plot of Long dan Xie gan pill samples (1-16 represents the number of chromatographic peaks in the common mode).

(3) Cluster Analysis. Hierarchical cluster analysis (HCA) is a conventional cluster analysis method. It is a detection tool that clearly reveals the natural grouping of data. The block distance was selected for distance calculation and HCA (Figure 8) was performed by error square sum method. Similar to the result of PCA, except that some samples from manufacturer $D$ are not distinguished from those of $A$, other samples from different enterprises could basically distinguish. Additionally, samples from B were relatively most far away from others. It indicated there existed some differences of these samples.

\subsection{Results of Multicomponent Quantification}

3.2.1. Linearity, $L O D$, and $L O Q$. Working standard solutions containing gentiopicrin, baicalin, baicalein, and wogonin were prepared by diluting the stock mixed solution with methanol to a series of proper concentrations. Then, they were injected and analyzed. The results of regression equations, linearity, determination coefficient, and limits of detection and quantification of the method are presented in
Table 3. The linear range varied from 3.25 to $492.56 \mu \mathrm{g} \mathrm{mL}^{-1}$, in accordance with the analyte. All analytes presented a determination coefficient $\left(R^{2}\right)$ of the 0.9999 , which allows the method to be considered linear. The limits of detection (LOD) and quantification (LOQ) were calculated according to guidelines for validation of analytical methods for pharmaceutical quality standards [22].

3.2.2. Instrument Precision. The same sample solution (no. 21) was injected for six consecutive times and analyzed. The RSDs of peak areas for gentiopicrin, baicalin, baicalein, and wogonin were $0.63 \%, 0.29 \%, 0.41 \%$, and $0.15 \%$, respectively. It indicated that the precision of the instrument was in accordance with the requirement in guidelines for validation of analytical methods for pharmaceutical quality standards [22].

3.2.3. Repeatability. The same batch of sample (no. 21) was taken and prepared for six independent sample solutions. Then, they were analyzed according to conditions under 2.6. 


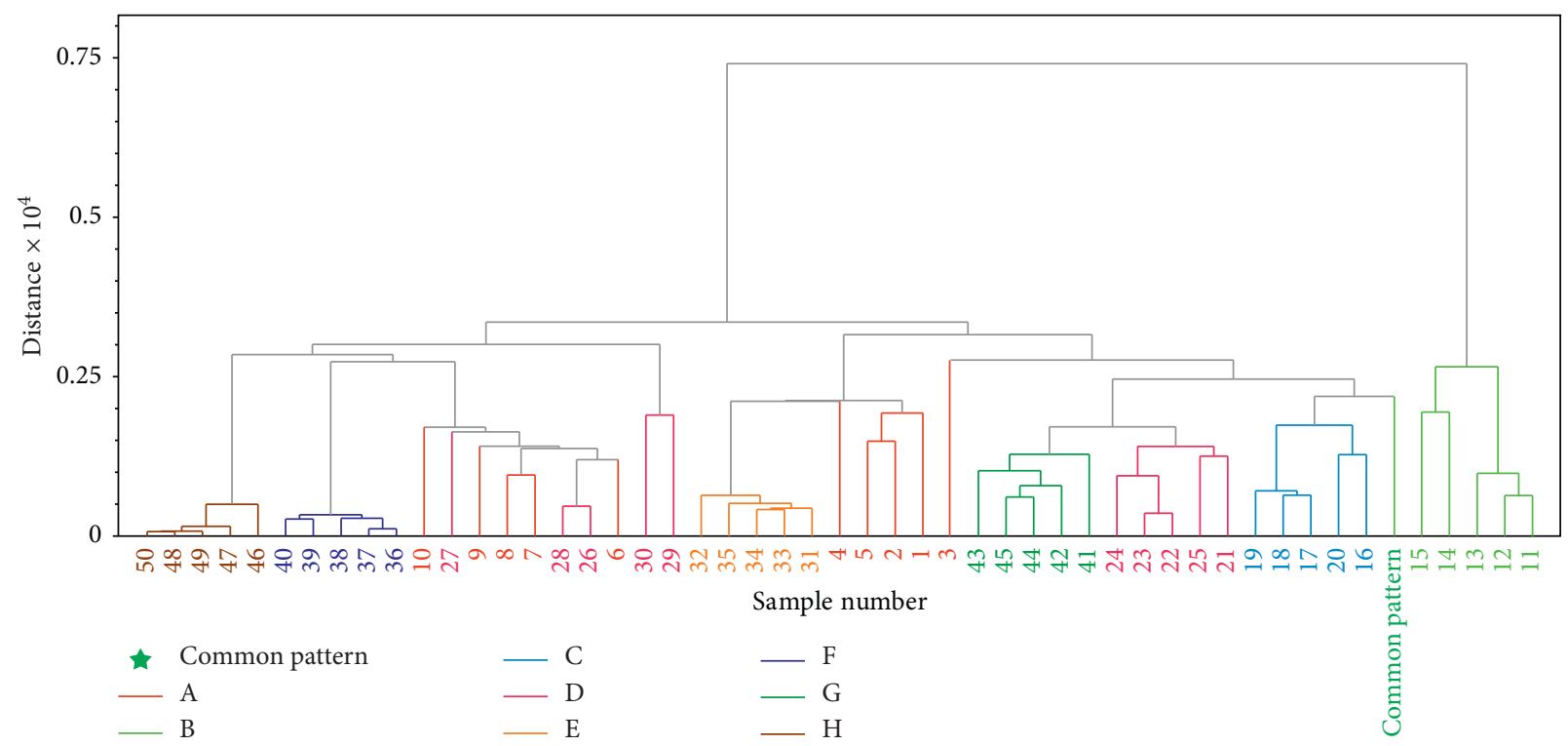

FIgURE 8: Dendrograms of hierarchical cluster analysis of Long dan Xie gan pill samples.

TABLE 3: Regression equations, linearity, determination coefficient, and limits of detection and quantification of the method.

\begin{tabular}{|c|c|c|c|c|c|}
\hline Components & $\begin{array}{c}\text { Regression } \\
\text { equations }\end{array}$ & $\begin{array}{c}\text { Linear } \\
\text { range } \\
\left(\mu \mathrm{g} \cdot \mathrm{mL}^{-1}\right)\end{array}$ & $R^{2}$ & $\begin{array}{l}\text { LOD } \\
\text { (ng) }\end{array}$ & $\begin{array}{c}\text { LOQ } \\
\text { (ng) }\end{array}$ \\
\hline $\mathrm{G}$ & $y=\begin{array}{r}11 \\
12\end{array}$ & $7.76 \sim$ & 0.9999 & 0.837 & 2.790 \\
\hline Baicalin & $\begin{array}{r}y=322 \\
425\end{array}$ & $7.88 \sim 492.56$ & 0.9999 & 0.431 & 1.077 \\
\hline Baic & $\begin{array}{r}y=43 \\
23\end{array}$ & $7.39 \sim 461.77$ & 0.9999 & 0.439 & 1.025 \\
\hline 0 & $\begin{array}{c}y=47996 x+ \\
23628\end{array}$ & $3.25 \sim 203.20$ & 0.9999 & 0.190 & 0.474 \\
\hline
\end{tabular}

The average contents of gentiopicrin, baicalin, baicalein, and wogonin were $4.77,3.84,0.62$, and $0.40 \mathrm{mg}^{-1} \mathrm{~g}^{-1}$, respectively. And the RSDs were $0.49 \%, 1.11 \%, 0.40 \%$, and $0.56 \%$, respectively. It indicated that method repeatability was in accordance with the requirement in guidelines for validation of analytical methods for pharmaceutical quality standards [22].

3.2.4. Stability. The same sample solution (no. 21) was injected at $0,4,8,12,18$, and $24 \mathrm{~h}$ at room temperature. The RSDs of contents for gentiopicrin, baicalin, baicalein, and wogonin were $1.88 \%, 1.54 \%, 1.70 \%$, and $3.22 \%$, respectively. It indicated that the sample solution was stable within $24 \mathrm{~h}$.

3.2.5. Recovery. The recovery experiment was performed by adding a known amount of individual reference standards into a certain amount of sample (no. 21).

Six separate samples of $1 \mathrm{~g}$ (contents of gentiopicrin, baicalin, baicalein, and wogonin were 4.77, 3.84, 0.62, and $0.40 \mathrm{mg} \cdot \mathrm{g}^{-1}$, respectively) were weighed accurately. And $25 \mathrm{~mL}$ of mixed reference standard solution (concentrations of gentiopicrin, baicalin, baicalein, and wogonin were $0.1861,0.1505,0.04856$, and $0.0300 \mathrm{mg} \cdot \mathrm{mL}^{-1}$, respectively) was added separately and prepared. The results (Table 4) showed that the average recoveries of four components ranged from $97.71 \%$ to $100.59 \%$ with RSDs in the range of $0.72 \%-1.29 \%$, which indicated that the method was accurate.

3.2.6. Sample Analysis. Fifty batches of sample solutions were prepared and analyzed. The results (Table 5) displayed that the contents of gentiopicrin, baicalin, baicalein, and wogonin were in the range of $0.61-5.40,1.96-5.33,0.10-$ 3.40 , and $0.046-1.16 \mathrm{mg} \cdot \mathrm{g}^{-1}$, respectively. It was easily to find the differences among samples from different enterprises by the scatter diagram (Figure 9). It showed that the general content trends of baicalin, baicalein, and wogonin were basically similar. Among them, the contents of baicalein and wogonin in samples from B were apparently higher than others; especially, the content of baicalein was much higher. The determination result was in accordance with the abovementioned PCA analysis result.

\subsection{Optimization of Experimental Conditions}

3.3.1. Investigation of Extraction Methods. The extraction method was optimized in order to make the fingerprint reflect the chemical composition information as much as possible. For both dosage forms of samples, different extraction solvent ( $80 \%$ methanol and 50\% methanol-water), and extraction mode and time (ultrasonic extraction for $30 \mathrm{~min}, 45 \mathrm{~min}$, and $60 \mathrm{~min}$ ) were investigated. The result showed that extraction time had little effect on both dosage forms. For WBP samples, the chromatogram could reflect rich chemical information with good separation of peaks with methanol extraction for $30 \mathrm{~min}$. While for $\mathrm{BCP}$ 
TABLE 4: Recovery results of four components in Long dan Xie gan pill samples.

\begin{tabular}{|c|c|c|c|c|c|c|c|}
\hline Components & No. & $\begin{array}{l}\text { Sampling amount } \\
\text { (g) }\end{array}$ & $\begin{array}{l}\text { Sample content } \\
(\mathrm{mg})\end{array}$ & $\begin{array}{l}\text { Added amount } \\
(\mathrm{mg})\end{array}$ & $\begin{array}{l}\text { Detected amount } \\
(\mathrm{mg})\end{array}$ & $\begin{array}{c}\text { Recovery } \\
(\%)\end{array}$ & $\begin{array}{c}\text { Average recovery } \\
(\%)\end{array}$ \\
\hline \multirow{6}{*}{ Gentiopicrin } & 1 & 1.0005 & 4.772 & 4.653 & 9.371 & 98.83 & \multirow{6}{*}{$\begin{array}{c}100.59 \% \text { (RSD } \\
1.22 \%)\end{array}$} \\
\hline & 2 & 1.0017 & 4.778 & 4.653 & 9.432 & 100.02 & \\
\hline & 3 & 1.0006 & 4.773 & 4.653 & 9.431 & 100.11 & \\
\hline & 4 & 1.0047 & 4.792 & 4.653 & 9.473 & 100.60 & \\
\hline & 5 & 1.0030 & 4.784 & 4.653 & 9.528 & 101.96 & \\
\hline & 6 & 1.0026 & 4.782 & 4.653 & 9.529 & 102.02 & \\
\hline \multirow{6}{*}{ Baicalin } & 1 & 1.0005 & 3.842 & 3.762 & 7.478 & 96.65 & \multirow{6}{*}{$\begin{array}{c}97.96 \%(\mathrm{RSD} \\
0.87 \%)\end{array}$} \\
\hline & 2 & 1.0017 & 3.847 & 3.762 & 7.537 & 98.09 & \\
\hline & 3 & 1.0006 & 3.842 & 3.762 & 7.503 & 97.32 & \\
\hline & 4 & 1.0047 & 3.858 & 3.762 & 7.583 & 99.02 & \\
\hline & 5 & 1.0030 & 3.852 & 3.762 & 7.543 & 98.11 & \\
\hline & 6 & 1.0026 & 3.850 & 3.762 & 7.554 & 98.56 & \\
\hline \multirow{6}{*}{ Baicalein } & 1 & 1.0005 & 0.620 & 1.214 & 1.807 & 97.78 & \multirow{6}{*}{$\begin{array}{c}97.71 \%(\mathrm{RSD} \\
0.72 \%)\end{array}$} \\
\hline & 2 & 1.0017 & 0.621 & 1.214 & 1.802 & 97.28 & \\
\hline & 3 & 1.0006 & 0.620 & 1.214 & 1.793 & 96.62 & \\
\hline & 4 & 1.0047 & 0.623 & 1.214 & 1.809 & 97.69 & \\
\hline & 5 & 1.0030 & 0.622 & 1.214 & 1.816 & 98.35 & \\
\hline & 6 & 1.0026 & 0.622 & 1.214 & 1.818 & 98.52 & \\
\hline \multirow{6}{*}{ Wogonin } & 1 & 1.0005 & 0.400 & 0.750 & 1.141 & 98.80 & \multirow{6}{*}{$\begin{array}{c}99.98 \%(\mathrm{RSD} \\
1.29 \%)\end{array}$} \\
\hline & 2 & 1.0017 & 0.401 & 0.750 & 1.146 & 99.33 & \\
\hline & 3 & 1.0006 & 0.400 & 0.750 & 1.141 & 98.80 & \\
\hline & 4 & 1.0047 & 0.402 & 0.750 & 1.153 & 100.13 & \\
\hline & 5 & 1.0030 & 0.401 & 0.750 & 1.156 & 100.67 & \\
\hline & 6 & 1.0026 & 0.401 & 0.750 & 1.167 & 102.13 & \\
\hline
\end{tabular}

TABLE 5: Contents of four components in Long dan Xie gan pills $\left(\mathrm{mg}^{-1} \mathrm{~g}^{-1}\right.$ ).

\begin{tabular}{|c|c|c|c|c|c|}
\hline No. & Manufacturers (dosage) & Gentiopicrin & Baicalin & Baicalein & Wogonin \\
\hline 1 & \multirow{5}{*}{$\mathrm{A}(\mathrm{WBP})$} & 1.579 & 3.191 & 0.778 & 0.433 \\
\hline 2 & & 1.391 & 2.912 & 0.992 & 0.449 \\
\hline 3 & & 3.295 & 3.102 & 0.741 & 0.429 \\
\hline 4 & & 1.801 & 2.582 & 0.718 & 0.381 \\
\hline 5 & & 1.884 & 3.006 & 0.976 & 0.526 \\
\hline 6 & \multirow{5}{*}{$\mathrm{A}(\mathrm{BCP})$} & 1.510 & 2.133 & 0.330 & 0.198 \\
\hline 7 & & 0.979 & 2.173 & 0.298 & 0.130 \\
\hline 8 & & 0.816 & 2.269 & 0.337 & 0.176 \\
\hline 9 & & 0.746 & 2.148 & 0.176 & 0.103 \\
\hline 10 & & 1.777 & 2.409 & 0.362 & 0.176 \\
\hline 11 & \multirow{5}{*}{$\mathrm{B}(\mathrm{WBP})$} & 1.019 & 4.153 & 3.049 & 1.129 \\
\hline 12 & & 1.013 & 4.394 & 3.141 & 1.158 \\
\hline 13 & & 0.963 & 3.763 & 3.280 & 1.080 \\
\hline 14 & & 0.860 & 4.396 & 3.133 & 0.994 \\
\hline 15 & & 0.826 & 5.330 & 3.405 & 1.084 \\
\hline 16 & \multirow{5}{*}{$\mathrm{C}(\mathrm{WBP})$} & 2.801 & 3.460 & 0.295 & 0.232 \\
\hline 17 & & 2.442 & 3.359 & 0.502 & 0.300 \\
\hline 18 & & 2.138 & 3.353 & 0.423 & 0.274 \\
\hline 19 & & 2.822 & 3.363 & 0.411 & 0.270 \\
\hline 20 & & 2.724 & 3.889 & 0.294 & 0.216 \\
\hline 21 & \multirow{5}{*}{$\mathrm{D}(\mathrm{WBP})$} & 4.753 & 3.747 & 0.622 & 0.406 \\
\hline 22 & & 4.166 & 3.123 & 0.570 & 0.367 \\
\hline 23 & & 4.344 & 3.118 & 0.590 & 0.381 \\
\hline 24 & & 4.028 & 3.047 & 0.585 & 0.385 \\
\hline 25 & & 4.084 & 3.642 & 0.589 & 0.390 \\
\hline 26 & \multirow{5}{*}{$\mathrm{D}(\mathrm{BCP})$} & 1.562 & 2.412 & 0.203 & 0.110 \\
\hline 27 & & 1.157 & 2.371 & 0.235 & 0.125 \\
\hline 28 & & 1.688 & 2.337 & 0.189 & 0.102 \\
\hline 29 & & 0.606 & 2.879 & 0.100 & 0.046 \\
\hline 30 & & 0.670 & 3.170 & 0.111 & 0.052 \\
\hline
\end{tabular}


TABLE 5: Continued.

\begin{tabular}{|c|c|c|c|c|c|}
\hline No. & Manufacturers (dosage) & Gentiopicrin & Baicalin & Baicalein & Wogonin \\
\hline 31 & & 1.922 & 2.210 & 0.722 & 0.314 \\
\hline 32 & & 1.620 & 2.205 & 0.698 & 0.324 \\
\hline 33 & $\mathrm{E}(\mathrm{WBP})$ & 1.815 & 2.304 & 0.715 & 0.323 \\
\hline 34 & & 1.749 & 2.188 & 0.715 & 0.320 \\
\hline 35 & & 1.650 & 2.407 & 0.779 & 0.333 \\
\hline 36 & & 0.690 & 2.297 & 0.817 & 0.387 \\
\hline 37 & & 0.669 & 2.279 & 0.837 & 0.397 \\
\hline 38 & F (WBP) & 0.675 & 2.175 & 0.811 & 0.385 \\
\hline 39 & & 0.640 & 2.051 & 0.800 & 0.374 \\
\hline 40 & & 0.660 & 1.963 & 0.821 & 0.394 \\
\hline 41 & & 4.544 & 4.012 & 0.587 & 0.291 \\
\hline 42 & & 5.331 & 3.771 & 0.524 & 0.256 \\
\hline 43 & G (WBP) & 5.103 & 3.419 & 0.505 & 0.263 \\
\hline 44 & & 5.402 & 3.990 & 0.567 & 0.261 \\
\hline 45 & & 5.128 & 3.862 & 0.501 & 0.249 \\
\hline 46 & & 2.822 & 3.082 & 0.372 & 0.176 \\
\hline 47 & & 2.768 & 3.214 & 0.376 & 0.181 \\
\hline 48 & $\mathrm{H}(\mathrm{BCP})$ & 2.721 & 3.177 & 0.367 & 0.178 \\
\hline 49 & & 2.736 & 3.177 & 0.365 & 0.180 \\
\hline 50 & & 2.758 & 3.153 & 0.359 & 0.168 \\
\hline
\end{tabular}

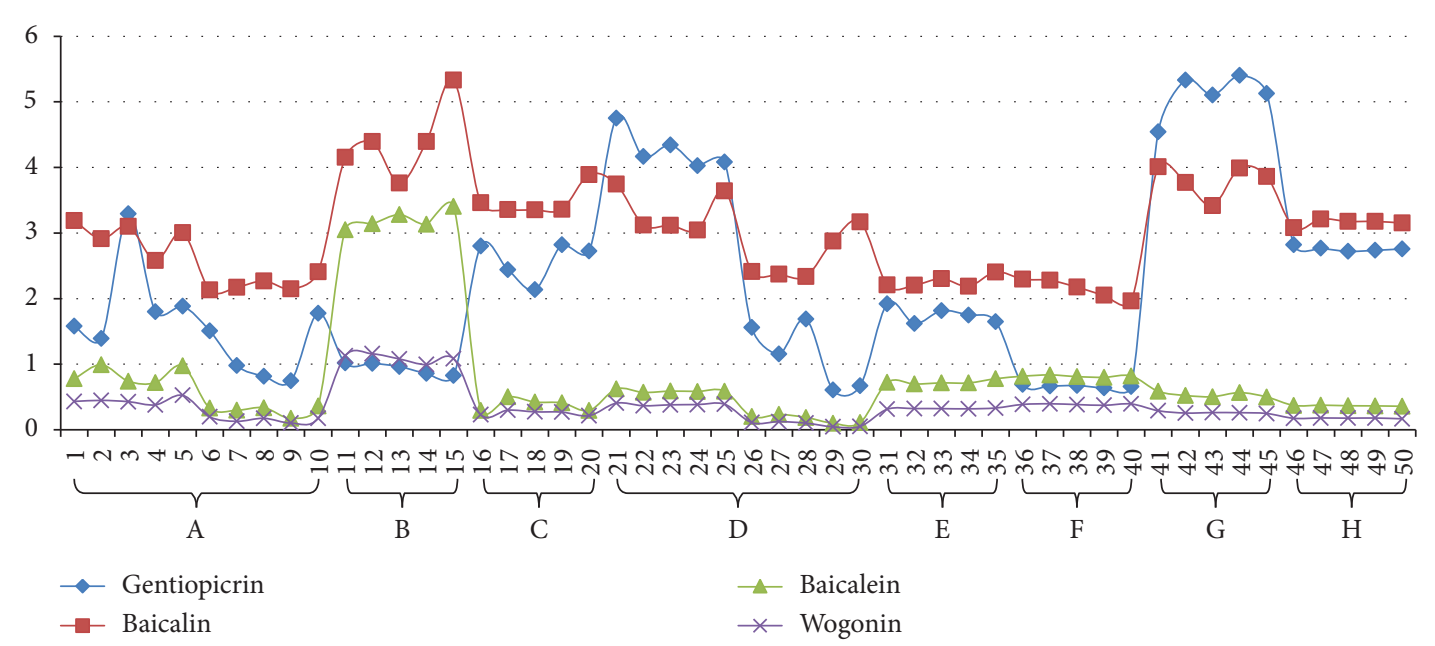

FIgURE 9: The scatter diagram of four components' contents in Long dan Xie gan pill samples.

samples, the dissolution was good by extraction with $80 \%$ methanol-water for $30 \mathrm{~min}$.

3.3.2. Study on the Chromatographic Condition. During the study, different mobile phase systems including methanolwater, acetonitrile-water, methanol- $0.1 \%$ formic acid, and acetonitrile- $0.1 \%$ formic acid were investigated. Also different chromatographic, columns including Waters Symmetry Shield ${ }^{\text {TM }}$ RP 18, Agilent Zorbax SB-C18, and Phenomenex Gemini C18 were experimented. Additionally, both DAD and DAD-ELSD detection were analyzed. As a result, the chromatogram was good on the Phenomenex Gemini C18 column with acetonitrile- $0.1 \%$ formic acid as the mobile phase under DAD detection. Finally, UV $240 \mathrm{~nm}$ was selected as detection wavelength for fingerprint establishment, since it could reflect much chemical information.
Meanwhile, UV $285 \mathrm{~nm}$ was determined as detection wavelength for simultaneous determination of main compounds due to the good separation.

\section{Conclusions}

Quality control is the key issue in modernization and internationalization of TCM. Qualitative fingerprint and quantitative multicomponent determination have been demonstrated as the comprehensive and effective way to accomplish the holistic quality analysis. In this study, both qualitative and quantitative works to get the overall composition and main components information were accomplished simultaneously by HPLC with dual-channel detection. Moreover, further deep mining of the data by chemometrics analysis helped to evaluate the quality of the 
preparation from different manufacturers. The result indicated that this approach is a powerful tool for quality control of TCM.

\section{Data Availability}

The data used to support the findings of this study are available from the corresponding author upon request.

\section{Conflicts of Interest}

The authors declare that there are no conflicts of interest regarding the publication of this paper.

\section{Acknowledgments}

The investigation was financially supported by the China Food and Drug Administration consignment inspection project.

\section{References}

[1] Chinese Pharmacopoeia Commission, Vol. 1, The Medicine Science and Technology Press of China, Beijing, China, 2015.

[2] Y. Jiang, B. David, P.-F. Tu, and Y. Barbin, "Recent analytical approaches in quality control of traditional Chinese medicine-a review," Analytica Chimica Acta, vol. 657, no. 1, pp. 9-18, 2010.

[3] H. Sun, X. Chen, A.-H. Zhang, T. Sakurai, J.-Z. Jiang, and X.-J. Wang, "Chromatographic fingerprinting analysis of Zhizhu Wan preparation by high-performance liquid chromatography coupled with photodiode array detector," Pharmacognosy Magazine, vol. 10, no. 40, pp. 470-476, 2014.

[4] C.-W. Chen, F.-Y. Song, H. Li, F. Chen, and C. Wang, "UPLC fingerprint and chemical pattern recognition method of Antai Pills," Chinese Journal of Pharmaceutical Analysis, vol. 36, no. 3, pp. 465-471, 2016.

[5] K.-X. Xu, Z.-N. Yin, W.-T. Zhang et al., "Study on UPLC fingerprint of Abri Herba," Chinese Journal of Pharmaceutical Analysis, vol. 38, no. 1, pp. 168-174, 2018.

[6] F.-Y. He, Y. He, X.-R. Hu, Z. Dai, and S.-C. Ma, "GC fingerprint analysis of dieda huoxue powder," Chinese Journal of Experimental Traditional Medical Formulae, vol. 23, no. 2, pp. 53-56, 2017.

[7] G.-X. Sun, Z.-F. Hou, W.-Y. Li, Y.-P. Yu, D.-D. Ma, and B. Chen, "Characteristics, composition and evaluation of multi-element and multi-dimensional fingerprints for traditional Chinese medicine," Central South Pharmacy, vol. 12, no. 6, pp. 497-504, 2014.

[8] C.-Y. Huang, D.-W. Ouyang, L.-X. Niu, J. Zhou, S. Lin, and $\mathrm{X}$. Hu, "Study on quality evaluation of dihuang (Rehmannia glutinosa) by two-dimension HPLC fingerprints and chemometrics methods," China Journal of Chinese Materia Medica, vol. 43, no. 8, pp. 1667-1674, 2018.

[9] M.-G. B. González, E.-P. Castaño, M.-S. Viñas, and D.-G. Evangelista, "Using the liquid-chromatographicfingerprint of sterols fraction to discriminate virgin olive from other edible oils," Journal of Chromatography A, vol. 1380, pp. 64-70, 2015.

[10] A. Hawrył, M. Hawrył, R. Świeboda, and M. W. Hajnos, "Chemometric analysis based on high-performance liquid chromatographic data in the fingerprint construction of selected Achillea species," Journal of Liquid Chromatography and Related Technologies, vol. 41, no. 1, pp. 1-5, 2017.

[11] A. Karioti, P. Timoteo, M.-C. Bergonzi, and A.-R. Bilia, "A validated method for the quality control of Andrographis paniculata preparations," Planta Medica, vol. 83, no. 14-15, pp. 1207-1213, 2017.

[12] J. Guan, H.-Y. Zhu, S.-Z. Yin et al., "Simultaneous determination of eight active components in Long Dan Xie Gan Pills by HPLC," Chinese Journal of Pharmaceutical Analysis, vol. 37, no. 12, pp. 2253-2259, 2017.

[13] K. Zan, C.-R. Wangjie, J. Lu, L.-N. Guo, J. Zheng, and S.-C. Ma, "Content determination of four diester diterpenoid alkaloids in leaves of Aconitum kusnezoffii by HPLC," China Journal of Chinese Materia Medica, vol. 43, no. 4, pp. 766-771, 2018.

[14] J.-D. Zhang, W. Guo, Y. Tang, A.-J. Shao, and B. Yang, "Quality assessment of commercial rosemary based on liquid/ gas chromatography and mass spectrometry with multivariate statistical analysis," China Journal of Chinese Materia Medica, vol. 43, no. 6, pp. 1192-1200, 2018.

[15] A.-P. Li, J.-J. Chen, Z.-Y. Li, and X.-M. Qin, "Quality consistency investigation of Astragali Radix based on ${ }^{1} \mathrm{H}-\mathrm{NMR}$ fingerprints," Journal of Chinese Medicinal Materials, vol. 40, no. 9, pp. 2107-2111, 2017.

[16] H. Liu, J. Su, X. Liang et al., "Identification and determination of the major constituents in traditional Chinese medicine Long Dan Xie Gan Pill by HPLC-DAD-ESI-MS," Journal of Pharmaceutical Analysis, vol. 1, no. 1, pp. 1-7, 2011.

[17] Q. Wang, W. Song, X. Qiao et al., "Simultaneous quantification of 50 bioactive compounds of the traditional Chinese medicine formula Gegen-Qinlian decoction using ultra-high performance liquid chromatography coupled with tandem mass spectrometry," Journal of Chromatography A, vol. 1454, no. 1, pp. 15-25, 2016.

[18] Y. Ma, J.-C. Li, W.-J. Huang et al., "Simultaneous determination of six triterpenoid acids from Guizhi Fuling capsules by UPLC-MS/MS," China Journal of Chinese Materia Medica, vol. 42, no. 17, pp. 3368-3373, 2017.

[19] M.-H. Yang, J.-S. Wang, and L.-Y. Kong, "Quantitative analysis of four major diterpenoids in Andrographis paniculata by ${ }^{1} \mathrm{H}$ NMR and its application for quality control of commercial preparations," Journal of Pharmaceutical and Biomedical Analysis, vol. 70, no. 1, pp. 87-93, 2012.

[20] M. W.-G. Wang, X.-L. Ma, X.-Y. Guo, M.-B. Zhao, P.-F. Tu, and Y. Jiang, "A series of strategies for solving the shortage of reference standards for multi-components determination of traditional Chinese medicine, Mahoniae Caulis as a case," Journal of Chromatography A, vol. 1412, no. 4, pp. 100-111, 2015.

[21] M.-H. Gad, E. Tuenter, N. Ei-Sawi et al., "Identification of some bioactive metabolites in a fractionated methanol extract from Ipomoea aquatic (Aerial parts) through TLC, HPLC, UPLC-ESI-QTOF-MS and LC-SPE-NMR fingerprints analyses," Phytochemical Analysis, vol. 29, no. 1, pp. 5-15, 2017.

[22] Chinese Pharmacopoeia Commission, Vol. 4, The Medicine Science and Technology Press of China, Beijing, China, 2015. 

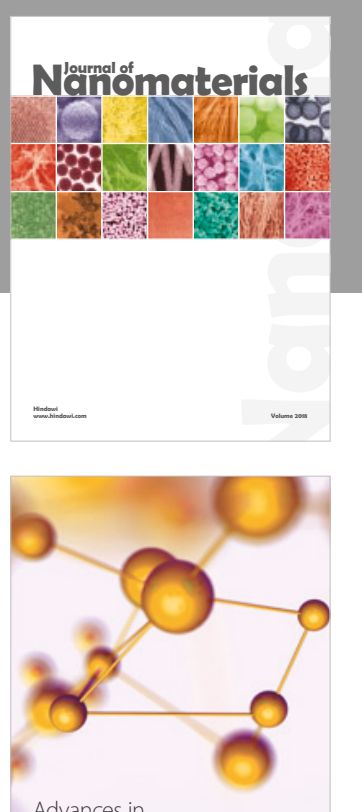

Physical Chemistry
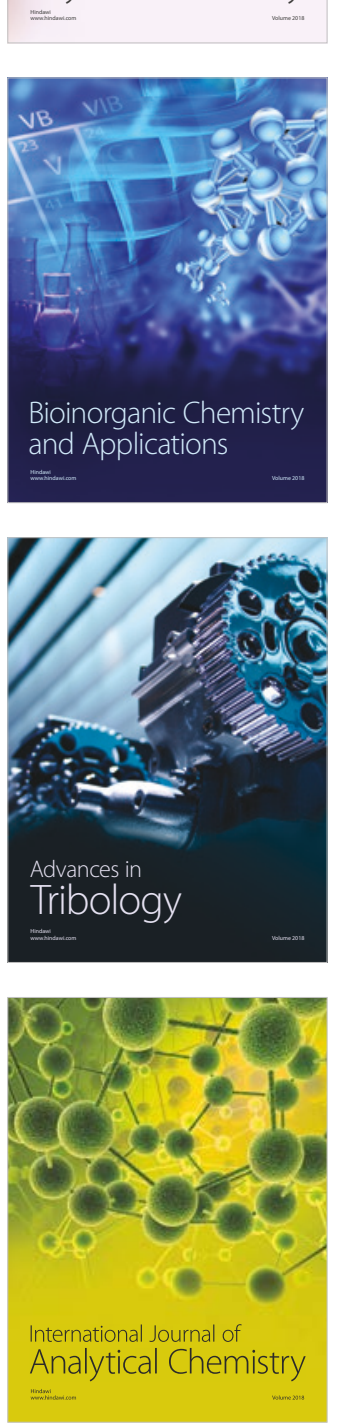

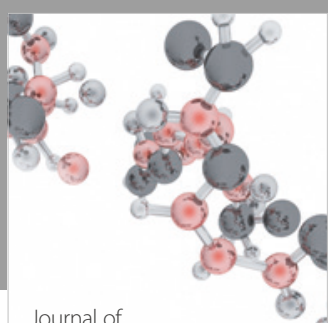

Analytical Methods

in Chemistry

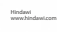

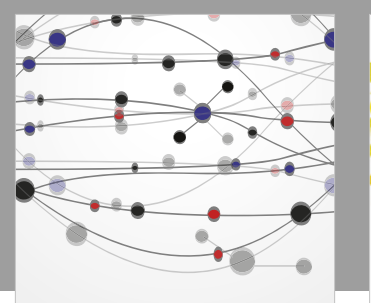

The Scientific World Journal

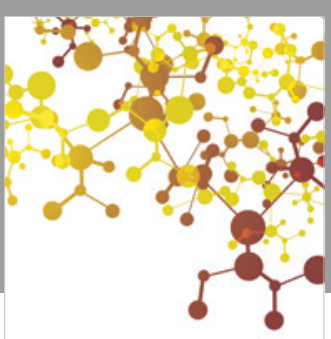

Journal of

Applied Chemistry
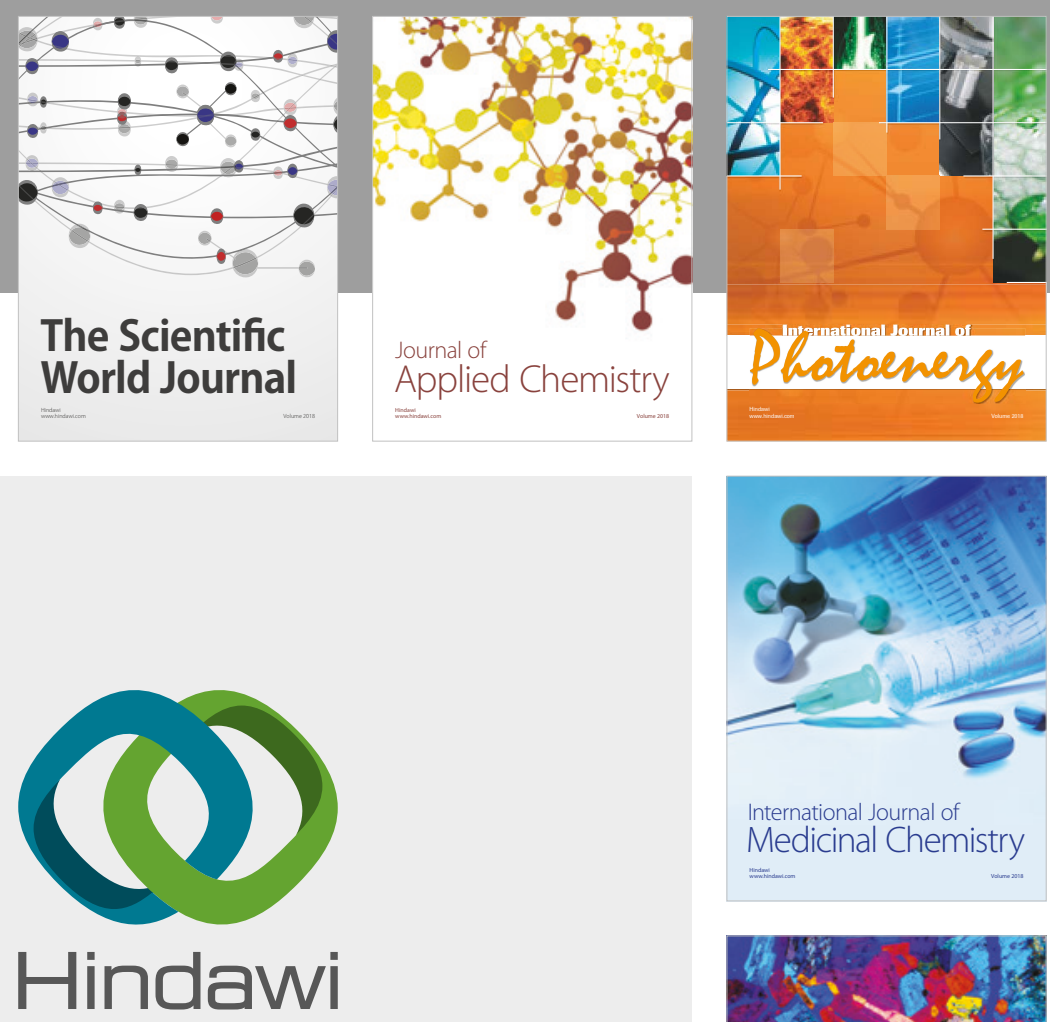

Submit your manuscripts at

www.hindawi.com
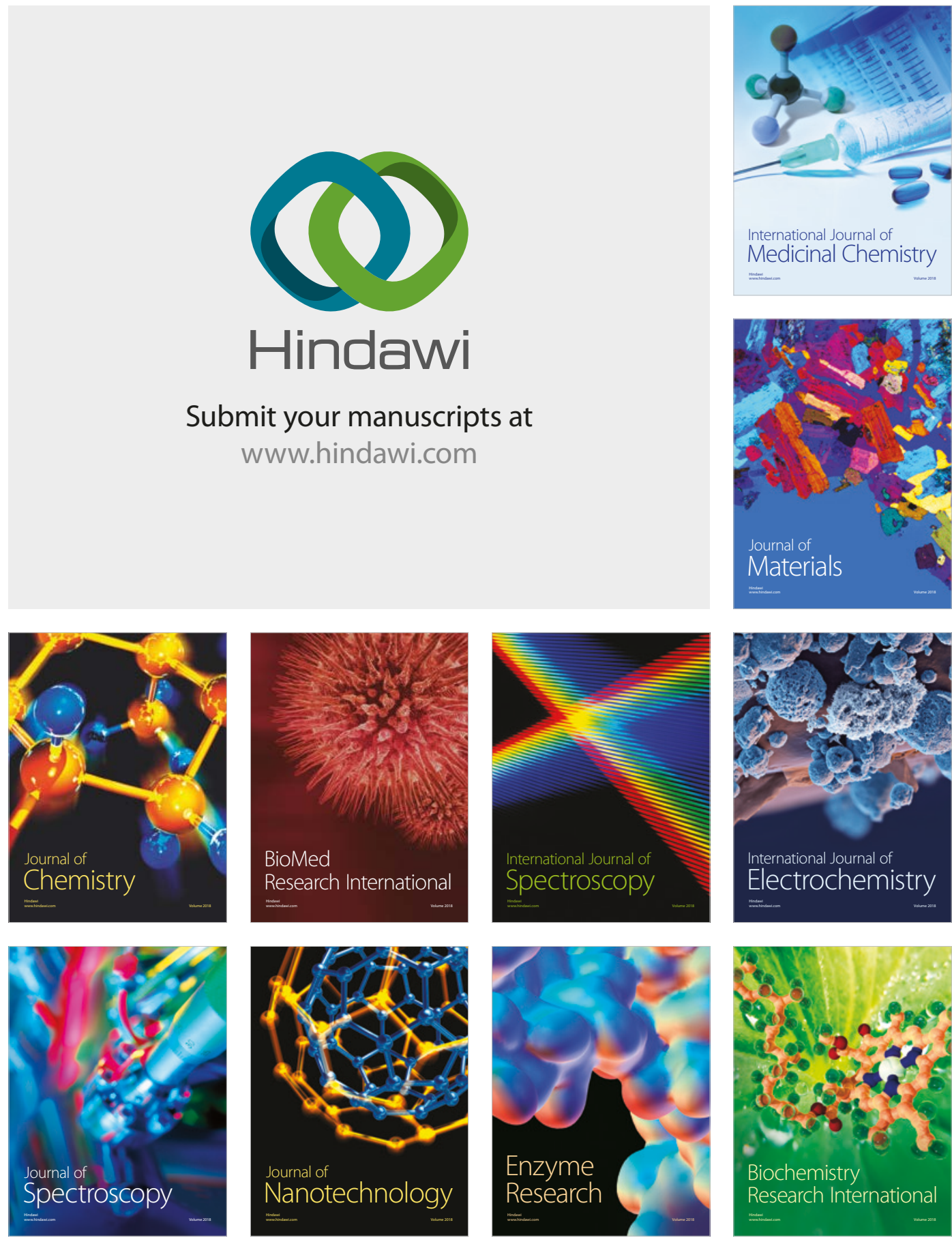
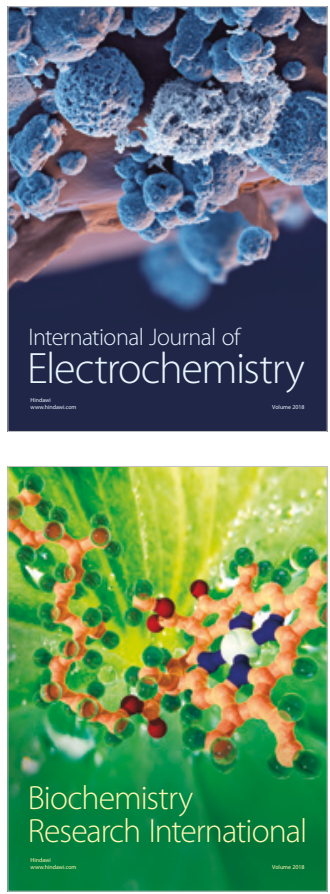